\title{
Tabagismo: abuso físico parental e fatores associados entre pacientes dos ambulatórios do Hospital Universitário Júlio Muller, Cuiabá (MT), 2013
}

\author{
Smoking: parental physical abuse and associated factors among \\ patients of the Júlio Muller University Hospital, Cuiabá (MT), 2013
}

\author{
Keyla Medeiros Maia Silva1, Delma Perpétua Oliveira de Souza²
}

\begin{abstract}
Resumo
Com o objetivo de analisar a associação do tabagismo e aspectos familiares, entre os quais o abuso físico parental, foi realizado um levantamento epidemiológico em 604 usuários dos ambulatórios do Hospital Universitário Júlio Müller da Universidade Federal de Mato Grosso, na faixa etária de 18 a 85 anos, de março a agosto de 2013. Aplicado questionário sobre os aspectos biossociodemográficos, familiares e tabagismo, sendo este definido conforme os critérios do Ministério da Saúde. Utilizou-se a análise bivariada, com teste do qui-quadrado e exato de Fisher e regressão de Poisson, com as medidas de associação de razão de prevalência com intervalo de confiança (IC) de 95\%. A prevalência de tabagismo foi de 18,2\%. No modelo final da análise observou-se que os fatores de exposição associados com significância estatística foram: abuso físico parental paterno, isto é, da figura do pai (RP 1,16; IC95\% 1,03-1,31), escolaridade (RP 1,13; IC95\% 1,06-1,20) e não ter os pais vivos (RP 1,12; IC95\% 1,05-1,20); e como fator de proteção, conviver com os pais toda a infância (RP 0,75; IC95\% 0,69-0,82) e ser da raça branca (RP 0,91; IC95\% 0,85-0,97). Os resultados indicam que a prevalência do tabagismo na população estudada está associada às relações estabelecidas na família. Sugere-se que a família seja considerada na implementação de ações educativas de prevenção.
\end{abstract}

Palavras-chave: tabagismo; relações familiares; epidemiologia; saúde coletiva.

\begin{abstract}
This study was done from March to August 2013 and examined the association between smoking and family aspects in a sample of 604 patients from the Júlio Muller University Hospital, Federal University of Mato Grosso in Cuiabá, Brazil. A questionnaire about bio-demographic, family history and smoking aspects was applied, which was defined according to the criteria of the Ministry of Health. Bivariate analysis with chi-square and Fisher's exact and Poisson regression was used with the association measures of prevalence ratios with confidence interval $(\mathrm{Cl})$ of $95 \%$. The prevalence of smoking was $18.2 \%$. It was observed that the exposure factors associated with statistic significance were: paternal physical abuse, ie, the father figure (PR 1.16; 95\% Cl 1.03-1.31), education (RP 1.13; 95\% Cl 1.06-1.20), and dead parents (RP 1.12; 95\% Cl 1.05-1.20); and as a protective factor to be white (PR 0.91; 95\% Cl 0.85-0.97) and living with parents (RP 0.75; 95\%Cl 0.69-0.82). Results indicate that the prevalence of smoking in the studied population is associated with established relationships in the family. This study suggests that family preventive educative actions must be carried in order to achieve changes in smoking behavior. Keywords: smoking; family relations; epidemiology; public health.
\end{abstract}

Trabalho realizado no Hospital Universitário Júlio Müller, Universidade Federal de Mato Grosso (UFMT) - Cuiabá (MT), Brasil.

'Mestranda do Instituto de Saúde Coletiva da UFMT - Cuiabá (MT), Brasil.

${ }_{2}^{2}$ Professora doutora do Instituto de Saúde Coletiva da UFMT - Cuiabá (MT), Brasil.

Endereço para correspondência: Keyla Medeiros Maia Silva - Hospital Universitário Júlio Müller, Ambulatório 2 - Pneumologia, UFMT - Rua Luís Philippe

Pereira Leite, s/n - Alvorada - CEP: 78048-902 - Cuiabá (MT), Brasil - E-mail: keyla_m@terra.com.br

Conflito de interesses: nada a declarar. 


\section{INTRODUÇÃO}

O tabagismo é a maior causa evitável de morte no mundo, apresentando-se como um problema de saúde pública desafiador ${ }^{1}$. O seu uso tem início na adolescência, em cerca de dois terços dos casos e, juntamente com o álcool, constituem as primeiras drogas na vida dos usuários de substâncias psicoativas ${ }^{2-4}$. Atualmente, estima-se a presença de 1,3 bilhão de pessoas que usam o tabaco fumado no mundo, consumindo cerca de 6 trilhões de unidades de cigarro/ano ${ }^{5}$. A Organização Mundial da Saúde (OMS) estima que um terço da população mundial maior de 15 anos seja fumante, com uma prevalência de $29 \%{ }^{6}$. Muitas são as doenças associadas ao tabaco, quer seja na sua forma ativa ou passiva, inclusive envolvendo a saúde infantil ${ }^{7,8}$.

A dependência química é um assunto de interesse mundial, com proporções epidêmicas. É definida como uma doença crônica ocasionada por alterações em várias regiões do cérebro, decorrente da ação prolongada da droga' ${ }^{9}$ É causada por várias substâncias, entre as quais, a nicotina. Trata-se de um assunto complexo que envolve um grande número de neurotransmissores e receptores cerebrais ${ }^{10}$.

Concebe-se a família como a unidade básica da sociedade, tendo quatro funções: sexual, reprodutiva, econômica e educativa ${ }^{11}$, sendo uma referência básica na formação de uma pessoa $^{12}$. Tomaremos como base teórica para entendermos a família a Teoria Sistêmica, que a vê como um sistema relacional, onde cada um dos seus componentes influencia o outro, assim como são influenciados por ele. Portanto, quando um dos seus membros apresenta sintomas, seja de depressão, ansiedade ou dependência química, a explicação e a solução não passa pela visão do indivíduo de forma isolada, e sim do ponto de vista de todo sistema relacional familiar. Essas interações ocorrem dentro de uma forma de organização que define a estrutura da família ${ }^{13}$.

Não existe uma definição clara entre uma família com desenvolvimento sadio, funcional e outra com desenvolvimento disfuncional, inclusive pelo fato das famílias funcionais passarem por fases disfuncionais, conforme seus vários estágios de vida ${ }^{14}$. Consideraremos funcional uma família que seja capaz de modificar sua estrutura conforme as circunstâncias, oferecendo condições para o desenvolvimento emocional adequado de seus membros,

apresentando a habilidade em mudar sua estrutura de poder, relações de papéis e regras de relacionamento em resposta a exigências situacionais ou de desenvolvimento ${ }^{15}$,

e famílias disfuncionais as que se tornam rígidas, não sendo capazes de fazer mudanças ${ }^{13,16}$.
Desta forma, o comportamento humano é visto essencialmente moldado por seu contexto social, relacional, sendo complexas as questões que envolvem o crescente uso das drogas em nossa sociedade, que vão muito além da visão anterior que contemplava o problema como só do indivíduo. Assim, a família passa a ser considerada coautora no processo da dependência química ${ }^{17,18}$.

A violência intrafamiliar é definida como

toda ação ou omissão que prejudique o bem-estar, a integridade física, psicológica ou a liberdade e o direito ao pleno desenvolvimento de um membro da família, podendo ser cometida dentro e fora de casa por qualquer integrante da família que esteja em relação de poder com a pessoa agredida ${ }^{19}$.

Neste estudo foi abordado o abuso físico parental, ou seja, os maus tratos por parte do pai e/ou da mãe, quer sejam biológicos ou não.

\section{METODOLOGIA}

\section{Tipo de estudo}

Foram utilizados dados de um levantamento epidemiológico com delineamento analítico de corte transversal, originalmente realizado para estudar tabagismo e fatores associados quanto a dados biossociodemográficos e características familiares (responsividade parental, negligência física, abuso sexual, abuso de substâncias parental e abuso físico parental).

\section{Local do estudo e população do estudo}

A amostra foi composta de pacientes maiores de 18 anos, atendidos nos vários ambulatórios do Hospital Universitário Júlio Muller, da Universidade Federal de Mato Grosso (UFMT), Cuiabá, de toda e qualquer especialidade médica.

\section{Amostra}

Amostragem por conveniência não probabilística, formada por pacientes presentes para atendimento em consulta, sendo convidados a participar da pesquisa até se atingir o tamanho da amostra, excluindo-se os que recusaram respondê-la, os que não estavam clinicamente em condições de responder as questões do instrumento, os que apresentaram dificuldade cognitivo-comportamental e os ex-fumantes.

Por desconhecer o número de pacientes atendidos nos ambulatórios do hospital, o tamanho da amostra foi calculado a partir da prevalência de tabagismo na cidade de Cuiabá, de $16 \%{ }^{20}$. O erro de amostragem foi de $3 \%$ com intervalo de confiança (IC) de $95 \%$ e $10 \%$ de perdas, obtendo um total de 660 pacientes. Destes, 56 foram excluídos por problemas no preenchimento do questionário, permanecendo 604 . 


\section{Coleta de dados}

Os entrevistadores eram de ambos os sexos, alunos de Medicina da UFMT e da Universidade de Cuiabá (UNIC), treinados pela pesquisadora. Os dados foram coletas de maio a agosto de 2013.

\section{Variáveis do estudo}

A variável dependente foi tabagismo, tomando como definição de fumante, não fumante e ex-fumante os critérios adotados pelo Ministério da Saúde ${ }^{20}$, a saber:

- Fumante: considerado fumante o indivíduo que responder positivamente à questão “ $\mathrm{O}$ (a) senhor(a) fuma?", independente do número de cigarros, da frequência e da duração do hábito de fumar.

- Não fumante: considerado não fumante o indivíduo que responder positivamente à questão " $\mathrm{O}(\mathrm{a})$ senhor(a) nunca fumou?"

- Ex-fumante: será considerado ex-fumante o indivíduo que responder positivamente à questão " $\mathrm{O}(\mathrm{a})$ senhor(a) já fumou?", independente do número de cigarros e da duração do hábito de fumar.

As variáveis independentes e suas categorizações foram: sexo (masculino, feminino), idade em anos (18-34 e 35-85), religião (sim/não), raça/cor conforme critérios do IBGE e categorizada (branca e outras), escolaridade (12 anos e mais, e 0 a 11 anos), convivência com os pais biológicos durante toda a infância (sim/não), pessoa mais importante no seu crescimento (pai e/ou mãe e outros), pais vivos (sim/não), pais casados (sim/ não), e abuso físico parental paterno (funcional/disfuncional) $\mathrm{e}$ abuso físico parental materno (funcional/disfuncional).

\section{Instrumentos de coleta de dados}

As informações foram coletadas por dois instrumentos: avaliação biossociodemográfica e o questionário de história familiar Family Background Questionnaire (FBQ-B) ${ }^{21}$. O FBQ-B é um instrumento para estimar o nível geral de funcionamento da família, composto por 68 perguntas distribuídas em oito subescalas, que abrangem diversas áreas do desenvolvimento humano. Quatro estudos foram realizados para verificação da fidedignidade e concordância deste instrumento ${ }^{22}$ :

1. Comparação entre população clínica e não clínica: estudo realizado com 265 participantes, obtendo-se um coeficiente Alpha de Cronbach de 0.99 para a escala total;

2. Análise Fatorial (população clínica e não clínica): estudo com 676 participantes. A avaliação da matriz correlacional sugeriu que o modelo fatorial obtido foi apropriado. Os itens que obtiveram coeficientes menores que 0.30 foram deletados, e o coeficiente Alpha de Cronbach para a escala total foi de 0.98 ;

3. Teste-reteste: estudo com 79 participantes. O coeficiente de correlação de Pearson entre os dois testes administrados foi, para a escala total, 0.96 ;

4. Comparação entre irmãos: estudo realizado com uma amostra de 35 pares de irmãos. O coeficiente de correlação de Pearson para a escala total entre os pares de irmãos foi 0.58 . Quando foram controladas as variáveis de socialização pelo gênero, incluindo somente pares de irmãos do mesmo sexo na análise $(\mathrm{n}=21)$, a correlação aumentou para 0.73 .

A versão do questionário utilizada em nosso estudo foi a reduzida, fornecida pelo autor e proveniente de um questionário maior, formando um subconjunto deste. $\mathrm{O}$ original é formado de 179 questões e 22 subescalas ${ }^{23,24}$, já validado no Brasi $^{25}$. Apesar de ser um questionário abrangente, sua grande extensão torna impraticável a aplicação para muitos propósitos, como em pesquisas como esta. Daí a aplicação da versão breve, construída pelo próprio autor, mantendo-se as subescalas que apresentam maior efeito sobre o desenvolvimento e funcionamento do indivíduo ${ }^{21}$.

Os dados foram avaliados numa escala Likert de 5 pontos, sendo que, quanto mais próximo de 5 , melhores níveis de funcionamento familiar e, quanto mais próximo de 1, pior. Para a pesquisa, foi considerada "disfuncional" a pontuação até 2,59, e "funcional" de 2,60 e mais. Este instrumento coleta informações da família de origem, verificando as recordações que o indivíduo tem a respeito dela, ocorridas até os seus 18 anos de idade. As subescalas utilizadas neste trabalho são "abuso físico parental, paterno e materno" que examinam um continuum disfuncional que inclui violência física e abuso.

\section{Teste e reteste}

A confiabilidade do instrumento foi avaliada pelo coeficiente de Kappa ${ }^{26}$, e a fidedignidade pelo coeficiente Alpha de Cronbrach ${ }^{27}$. A interpretação foi feita por Landis e $\mathrm{Koch}^{28}$, sendo realizado o teste-reteste após sete dias, em 14 pacientes. Obtida média de 0,63 no Kappa, correspondendo a boa concordância, e de 0,80 no Cronbach, considerada alta fidedignidade.

\section{Análise dos dados}

O armazenamento dos dados foi feito pelo programa Epi-Info 2000, versão 6.0, e a análise estatística pelo programa SPSS versão 15 , e Stata versão 10 . A análise estatística foi realizada em duas etapas: 1. Bivariada, onde foi feita a associação da variável dependente tabagismo com todas as varáveis independentes. A medida de associação utilizada foi a razão de prevalência (RP) com os respectivos intervalos de 
confiança (IC95\%). O controle de variáveis de confusão e de interação (modificadora de efeito) foi realizado por regressão de Poisson. Nesse modelo entraram todas as variáveis que na análise bivariada apresentaram um valor $\mathrm{p}$ (significância)

Tabela 1. Distribuição segundo características biossociodemográficas e familiares obtidas pela análise univariada dos pacientes dos ambulatórios do Hospital Universitário Júlio Müller, 2013

\begin{tabular}{|c|c|c|c|}
\hline Variável & Categoria & $\mathbf{n}$ & $\%$ \\
\hline \multirow[t]{2}{*}{ Sexo } & Feminino & 499 & 74,5 \\
\hline & Masculino & 172 & 25,5 \\
\hline \multirow[t]{2}{*}{ Faixa etária (anos) } & 18-34 anos & 183 & 30,3 \\
\hline & $35-85$ anos & 421 & 69,7 \\
\hline \multirow[t]{2}{*}{ Raça/Cor } & Branca & 182 & 30,1 \\
\hline & Outras & 422 & 69,9 \\
\hline \multirow[t]{2}{*}{ Religião } & $\operatorname{Sim}$ & 583 & 96,5 \\
\hline & Não & 21 & 3,5 \\
\hline \multirow[t]{2}{*}{ Escolaridade } & 12 e mais & 182 & 30,1 \\
\hline & $0-11$ anos & 422 & 69,9 \\
\hline \multirow{2}{*}{$\begin{array}{l}\text { Conviveu com os pais } \\
\text { durante toda a infância }\end{array}$} & Sim & 453 & 75,0 \\
\hline & Não & 151 & 25,0 \\
\hline \multirow{2}{*}{$\begin{array}{l}\text { Pessoa mais importante } \\
\text { em seu crescimento }\end{array}$} & Pai e/ou Mãe & 550 & 91,1 \\
\hline & Outros & 54 & 8,9 \\
\hline \multirow[t]{2}{*}{ Pais vivos } & Sim & 229 & 37,9 \\
\hline & Não & 375 & 62,1 \\
\hline \multirow[t]{2}{*}{ Pais casados } & Sim & 462 & 76,5 \\
\hline & Não & 142 & 23,5 \\
\hline \multirow[t]{2}{*}{ Abuso físico paterno } & Funcional & 527 & 87,3 \\
\hline & Disfuncional & 77 & 12,7 \\
\hline \multirow[t]{2}{*}{ Abuso físico materno } & Funcional & 583 & 96,5 \\
\hline & Disfuncional & 21 & 3,5 \\
\hline
\end{tabular}

Percentagem ponderada (\%); Raça/Cor: conforme classificação IBGE, categorizada

Tabela 2. Associação do tabagismo segundo características biossociodemográficas obtidas pela análise bivariada entre os pacientes dos ambulatórios do Hospital Universitário Júlio Müller, 2013

\begin{tabular}{lcccc}
\hline & \multicolumn{2}{c}{ Tabagismo } & \multicolumn{2}{c}{ Análise bruta } \\
\cline { 2 - 3 } Variável & Sim & Não & RP (IC95\%) & Valor $\mathbf{p}^{*}$ \\
\cline { 2 - 3 } & $\mathbf{n}(\%)$ & $\mathbf{n}(\%)$ & & \\
\hline Faixa etária & & & \\
18-34 anos & $19(10,38)$ & $164(89,62)$ & 1,00 & \\
35-85 anos & $93(22,09)$ & $328(77,91)$ & $1,24(1,12-1,38)$ & 0,0007 \\
Sexo & & & & \\
Feminino & $69(15,33)$ & $381(84,67)$ & 1,00 & \\
Masculino & $43(27,92)$ & $111(72,08)$ & $1,70(1,27-2,26)$ & 0,0005 \\
Religião & & & & \\
Sim & $104(17,80)$ & $481(82,20)$ & 1,00 & \\
Não & $8(42,10)$ & $11(57,90)$ & $3,19(1,31-7,75)$ & 0,0072 \\
Raça/Cor & & & & \\
Branca & $32(17,58)$ & $150(82,42)$ & $0,94(0.68-1,29)$ & 0,7086 \\
Outras & $80(19,04)$ & $344(81,90)$ & 1,00 & \\
Escolaridade & & & & \\
12 a e + & $19(10,44)$ & $163(89,56)$ & 1,00 & \\
0 -11 a & $93(22,04)$ & $329(77,96)$ & $1,24(1,11-1,37)$ & 0,0008
\end{tabular}

Percentagem ponderada; RP: Razão de Prevalência; IC95\%: Intervalo confiança de $95 \%$; *valor significante do teste de associação do $\chi^{2}$ igual ou menor que 0,25 . Iniciou-se por modelo saturado com a variável de exposição e todas as possíveis variáveis de confusão e termos de interação. $\mathrm{Na}$ análise final permaneceram as variáveis com $p<0,05$, por serem estas as com significância estatística ${ }^{29-31}$.

\section{Questões éticas}

Projeto aprovado pelo Comitê de Ética e Pesquisa do Hospital Universitário Júlio Müller da UFMT (parecer $n^{\circ}$ 146.506/CEP/HUJM/2012).

\section{RESULTADOS}

A amostra foi composta por 604 pacientes de ambos os sexos, sendo 450 mulheres $(74,5 \%)$, com a média de idade de 43,89 (DP=14,07).

A Tabela 1 apresenta os dados da análise univariada, dos quais destacamos que, quanto ao grau de escolaridade, $69,9 \%$ dos pacientes apresentavam de 0 a 11 anos de estudo, e $30,1 \%$ anotavam 12 anos e mais; $75 \%$ dos pacientes referiram ter convivido com os pais biológicos toda a infância, sendo que $91,1 \%$ consideraram ser a mãe e/ou o pai a figura mais importante, e 8,9\% relataram ser "outros" a figura mais importante em seu crescimento; 37,9\% dos pacientes referiram que os pais ainda eram vivos, e $76,5 \%$ referiam que os pais eram casados. Com relação ao abuso físico parental paterno, $12,7 \%$ apresentaram disfunção familiar nesta área, enquanto na avaliação do abuso físico parental materno, apenas 3,5\% o apresentaram.

A Tabela 2 apresenta os dados de associação entre tabagismo e as variáveis biossociodemográficas. A prevalência de tabagismo variou com a idade, gênero e escolaridade. As variáveis biossociodemográficas que apresentaram associação positiva e estatisticamente significativa pela análise bivariada foram: idade, sexo, escolaridade e religião. A Tabela 3 apresenta os dados de associação entre tabagismo e as variáveis familiares. As variáveis familiares que apresentaram associação positiva e estatisticamente significativa pela análise bivariada foram: conviveu com os pais toda a infância, pessoa mais importante durante seu crescimento, pais casados e abuso físico parental paterno. A Tabela 4 apresenta os resultados da análise multivariada por regressão de Poisson. Verificou-se que as variáveis associadas ao desfecho tabagismo foram: escolaridade, pais vivos e abuso físico paterno.

\section{DISCUSSÃO}

A violência intrafamiliar contra a criança e o adolescente é um problema de grande envergadura, sendo universal e 
Tabela 3. Associação do tabagismo segundo características familiares obtidas pela análise bivariada entre os pacientes dos ambulatórios do Hospital Universitário Júlio Müller, 2013

\begin{tabular}{|c|c|c|c|c|}
\hline \multirow{2}{*}{ Variável } & \multicolumn{2}{|c|}{ Tabagismo } & \multicolumn{2}{|c|}{ Análise bruta } \\
\hline & Sim & Não & RP (IC95\%) & Valor $\mathrm{p}^{*}$ \\
\hline \multicolumn{5}{|c|}{ Conviveu com os pais toda a infância? } \\
\hline Sim & $74(16,34)$ & $379(83,66)$ & 1 & \\
\hline Não & $38(25,17)$ & $113(74,83)$ & $1,47(1,08-2,00)$ & 0,0156 \\
\hline \multicolumn{5}{|c|}{ Pessoa mais importante durante seu crescimento } \\
\hline Pai e/ou Mãe & $94(17,09)$ & $456(82,90)$ & 1 & \\
\hline Outros & $18(33,33)$ & $36(66,67)$ & $2,19(1,29-3,72)$ & 0,0034 \\
\hline \multicolumn{5}{|l|}{ Pais vivos } \\
\hline Sim & $35(15,28)$ & $194(84,72)$ & 1 & \\
\hline Não & $77(20,53)$ & $298(79,47)$ & $1,13(0,98-1,31)$ & 0,1073 \\
\hline \multicolumn{5}{|l|}{ Pais casados } \\
\hline Sim & $76(16,45)$ & $386(83,55)$ & 1 & \\
\hline Não & $36(25,35)$ & $106(74,65)$ & $1,49(1,08-2,04)$ & 0,0170 \\
\hline \multicolumn{5}{|c|}{ Abuso físico parental paterno } \\
\hline Não & $91(17,27)$ & $436(82,73)$ & 1 & \\
\hline Sim & $21(27,27)$ & $56(72,73)$ & $1,64(1,04-2,60)$ & 0,0349 \\
\hline \multicolumn{5}{|c|}{ Abuso físico parental materno } \\
\hline Não & $108(18,52)$ & $475(81,46)$ & 1 & \\
\hline Sim & $4(19,05)$ & $17(80,95)$ & $1,03(0,35-3,01)$ & 0,9517 \\
\hline
\end{tabular}

Percentagem ponderada; RP: Razão de prevalência; IC95\%: Intervalo confiança de 95\%; *valor significativo do teste de associação do $\chi^{2}$

endêmico, ocorrendo em várias culturas, etnias, sexo, classes sociais e poder econômico. Apesar desta amplitude, encontra na pobreza um catalisador ${ }^{32}$. No dia a dia, há uma tendência a minimizar os danos por ela causados, em nome da disciplina, não havendo consenso quanto ao método que se considera violento no processo educacional. Porém, a tendência mundial é considerar violência física doméstica contra a criança ou o adolescente qualquer ato disciplinar que atinja seu corpo.

Existem quatro maneiras de exercer a violência doméstica: física, psicológica, negligência e sexual. Neste artigo estaremos abordando o abuso físico exercido pelo pai e/ou mãe, de grande frequência e que tem muita influência no ajustamento psicológico e desenvolvimento da personalidade, tendo o Fourth National Incidence Study of Child Abuse and Neglect mostrado que a $64 \%$ dos maus-tratos ocorrem dentro da família e pelos pais biológicos ${ }^{33}$.

A violência física é a forma mais frequente de violência domiciliar, variando de uma palmada a espancamentos e homicídios. Seus efeitos reduzem as chances de um desenvolvimento integral e saudável, favorecendo o uso e abuso de substâncias psicoativas como álcool, tabaco e outras drogas, prática de sexo inseguro, gestação na adolescência, baixa autoestima e culpa, distúrbios alimentares com obesidade e distúrbios psiquiátricos ${ }^{4,32}$.

A dependência química (DQ) manifesta-se clinicamente como uma síndrome, isto é, um conjunto de sinais e sintomas, podendo ser causada por várias substâncias: álcool; nicotina; maconha; cocaína e crack; benzodiazepínicos, hipnóticos e ansiolíticos; opioides; anfetaminas e metanfetaminas; alucinógenos; anabolizantes e inalantes e outras drogas (cetamina, ácido y-hidroxibutirato (GHB), cloridrato de bezidamina,
Tabela 4. Associação do tabagismo segundo características biossociodemográficas e familiares obtidas mediante análise multivariada por regressão de Poisson entre os pacientes dos ambulatórios do Hospital Universitário Júlio Müller, 2013

\begin{tabular}{|c|c|c|}
\hline \multirow[t]{2}{*}{ Variável } & \multicolumn{2}{|c|}{$\begin{array}{c}\text { Análise multivariada } \\
\text { ajustada }^{\star}\end{array}$} \\
\hline & RP (IC95\%) & Valor $\mathrm{p}^{\star}$ \\
\hline \multicolumn{3}{|c|}{ Faixa etária (anos) } \\
\hline 18-34 anos & 1 & \\
\hline $35-85$ anos & $0,98(0,92-1,06)$ & 0,734 \\
\hline \multicolumn{3}{|l|}{ Sexo } \\
\hline Feminino & 1 & \\
\hline Masculino & $0,95(0,88-1,02)$ & 0,182 \\
\hline \multicolumn{3}{|l|}{ Religião } \\
\hline Sim & 1 & \\
\hline Não & $1,11(0,91-1,34)$ & 0,288 \\
\hline \multicolumn{3}{|l|}{ Raça } \\
\hline Branca & 1 & \\
\hline Outras & $0,90(0,85-0,97)$ & 0,006 \\
\hline \multicolumn{3}{|l|}{ Escolaridade } \\
\hline 12 anos e mais & 1 & \\
\hline $0-11$ anos & $1,13(1,06-1,20)$ & 0,000 \\
\hline \multicolumn{3}{|c|}{ Conviveu com os pais toda a infância? } \\
\hline Sim & 1 & \\
\hline Não & $0,75(0,69-0,82)$ & 0,000 \\
\hline \multicolumn{3}{|c|}{$\begin{array}{l}\text { Pessoa mais importante em seu } \\
\text { crescimento }\end{array}$} \\
\hline Pai e/ou Mãe & 1 & \\
\hline Outros & $1,02(0,90-1,15)$ & 0,725 \\
\hline \multicolumn{3}{|l|}{ Pais vivos } \\
\hline Sim & 1 & \\
\hline Não & $1,15(1,08-1,22)$ & 0,000 \\
\hline \multicolumn{3}{|c|}{ Abuso físico paterno } \\
\hline Funcional & 1 & \\
\hline Disfuncional & $1,16(1,03-1,31)$ & 0,010 \\
\hline \multicolumn{3}{|c|}{ Abuso físico materno } \\
\hline Funcional & 1 & \\
\hline Disfuncional & $0,98(0,82-1,16)$ & 0,831 \\
\hline
\end{tabular}


chá de fita, cápsula de vento, cafeína e efedrina) ${ }^{34}$. Inúmeros estudos mostram que a nicotina preenche os critérios para ser definida como droga que traz dependência. Estes critérios foram estabelecidos pela Associação Americana de Psiquiatria por meio do documento conhecido como DMS-IV, 1994³. Porém, apesar da nicotina ser uma droga psicoativa que traz dependência, é de uso legal e de fácil acesso por sua ampla comercialização.

Pouco de sabe a respeito do cenário familiar dos seus adictos, enquanto o funcionamento de famílias com membros dependentes de outras substâncias psicoativas é mais conhecido. Fazendo um paralelo com o que já conhecemos da família com problemas de adicção, observa-se que nesta o pai tende a ter uma relação de ausência, de desapego emocional com os filhos, com um modelo fraco de masculinidade, mas associado a uma disciplina dura, incoerente com o vínculo emocional, estabelecendo relações difíceis, caracterizadas pelo distanciamento emocional e os maus-tratos ${ }^{17,36}$. Essa associação foi encontrada em nosso estudo, com significância estatística, mediante os resultados da variável abuso físico parental paterno, conforme Tabelas 3 e 4. O mesmo não foi observado na variável abuso físico parental materno. Tal resultado pode expressar outra característica da família com dependência química, qual seja a mãe do dependente com tendência a relacionar-se com ele de forma superprotetora, criando-o num ambiente de limites pouco claros e frouxos ${ }^{17}$.

Outra associação com valor estatisticamente significativo na análise multivariada foi a ausência de um dos pais ${ }^{37}$. Em nosso estudo encontramos prevalência de tabagismo de $15,28 \%$ quando pais vivos, e de 20,53\% quando não, RP 1,15 , IC95\% 1,08-1,22, valor p 0,000.

Além destas associações, encontramos significância estatística com relação a escolaridade, sendo menor a prevalência conforme aumento do número de anos estudados, compatível com os achados nacionais ${ }^{38}$.

\section{CONCLUSÃO}

$\mathrm{O}$ tabagismo é considerado uma dependência química $\mathrm{e}$ psicológica advinda do hábito apreendido pela repetição, de início na infância e adolescência, causadora de milhares de mortes em todo o mundo.

Este estudo verificou que vários são os fatores a ele associados, entre os quais o abuso físico parental paterno. Este ocorre de forma velada no seio familiar, somente a vitima sabendo a dor sentida. Sua associação com o tabagismo sugere a realização de outros estudos com o foco nas relações familiares, que possam subsidiar ações de prevenção e tratamento.

\section{REFERÊNCIAS}

1. World Health Organization. WHO report on the global tobacco epidemic, 2008: The MPOWER package. Geneva: WHO; 2008.

2. Malcon MC, Menezes AMB, Chartkin M. Prevalência e fatores de risco para tabagismo em adolescentes: estudo de base populacional no Sul do Brasil. Rev Saúde Pública. 2003;37(1):1-7.

3. Myers MG, Kelly FJ. Cigarette smoking among adolescents with alcohol and other drug use problems. Alcohol Res Health. 2006;29(3):221-27.

4. Jesus FB, Lima FCA, Martins CBG, Souza SPS. Vulnerabilidade na adolescência: a experiência e expressão do adolescente. Rev Gaúcha Enferm. 2011;32(2):359-67.

5. World Health Organization. Global Status Report on Alcohol and Health, 2011. Geneva: WHO; 2011.

6. World Health Organization \& International Union against Tuberculosis and Lung Diseases. Monograph on TB and tobacco control: joining efforts to control two related global epidemics. Geneva: WHO; 2007.

7. World Health Organization. Tobacco \& the Rights of the Child. Geneva: WHO; 2001.

8. Bettiol H, Barbieri MA, Silva AAM. Epidemiologia do nascimento prétermo: tendências atuais. Rev Bras Ginecol Obstet. 2010;32(2):57-60.

9. Silva CJ, Laranjeira RR. Neurobiologia da dependência química. Cap. 2, Aconselhamento em dependência química. 2a ed. São Paulo: Roca; 2010.
10. Haggsträm FM, Chatkin JM. Dependência à nicotina. Tabagismo: do diagnóstico à Saúde Pública. Atheneu. 2007;3:25-31.

11. Osório LC. Família hoje. Porto Alegre: Artemed; 1996.

12. Silva EA. As redes comunitárias e de saúde no atendimento aos usuários e dependentes de substâncias psicoativas - módulo 6. Coordenação do módulo Marcelo Santos Cruz. Brasília: Secretaria Nacional de Políticas sobre Drogas; 2008.

13. Nichols MP, Schwartz RC. Terapia familiar: conceitos e métodos. $7^{\mathrm{a}} \mathrm{ed}$. Porto Alegre: Artmed; 2007.

14. Carter B, McGoldrick M. As mudanças no ciclo de vida familiar: uma estrutura para a terapia familiar. 2a ed. Porto Alegre: Artes Médicas; 2001.

15. Olson DH. Circumplex Model VII: Validation studies and faces III. Family Process. 1986;25:337-51.

16. Szymanski H. Práticas educativas familiares: a família como foco de atenção psicoeducacional. Estud Psicol (Campinas). 2004;21(2):5-16.

17. Orth APS, Moré CLOO. Funcionamento de famílias com membros dependentes de substâncias psicoativas. Psicol Argum. 2008;26(55):293-303.

18. Schenker M, Minayo MCS. A importância da família no tratamento do uso abusivo de drogas: uma revisão da literatura. Cad Saúde Pública. 2005;20(3):649-59.

19. Brasil. Ministério da Saúde. Secretaria de Políticas de Saúde. Violência intradomiciliar: orientações para práticas em serviços. Brasília: MS; 2001. 
20. VIGITEL, 2011. Vigilância de fatores de risco e proteção para as doenças crônicas por inquérito telefônico. SUS/ Ministério da Saúde; 2012.

21. Melchert TP, Kalemeera A. A brief version of the Family Background Questionnaire. Measurement and Evaluation in Counseling and Development. 2009;41:210-22.

22. Melchert TP, Sayger TV. The development of an instrument for measuring memories of family of origin characteristics. Educational and Psychological Measurement. 1998;58(1):99-118.

23. Melchert TP. Testing the validity of an instrument for assessing family of origin history. J Clin Psychol. 1998;54(7):863-76.

24. Melchert TP. A review of instruments for assessing family history. Clin Psychol Rev. 1998;18(2):163-87.

25. Falcke D. Águas passadas não movem moinhos? As experiências na família de origem como preditoras da qualidade do relacionamento conjugal [tese doutorado]. Porto Alegre: Universidade Católica do Rio Grande do Sul (PUCRS), Curso de Psicologia; 2003.

26. Cohen JA. Coefficient of agreement for nominal scales. Educacional and Psychological Measurement. 1960;20:37-46.

27. Freitas ALP, Rodrigues SG. A avaliação da confiabilidade de questionários: uma análise utilizando o coeficiente alfa de Cronbach. XII SIMPEP. 2003 Nov 7-9; Bauru (SP), Brasil.

28. Landis JR, Koch GG. The measurement of observer agreement for categorical data. Biometric. 1977;33(1):159-74.

29. Barros A, Hirakata V. Alternatives for logistic regression in crosssectional studies: an empirical comparison of models that directly estimate the prevalence ratio. BMC Med Res Methodol. 2003;3:21.
30. Francisco P, Donalisio M, Barros M, Cesar C, Carandina L, Goldbaum M. Association measures in cross-sectional studies with complex samplings: odds ratio and prevalence ratio. Rev Bras Epidemiol. 2008;11:347-55.

31. Skov T, Deddens J, Petersen M, Endaht L. Prevalence proportion ratios: estimation and hypothesis testing. Int J Epidemiol. 1998;27:91-5.

32. Day VP, Telles LEB, Zoratto PH, Azambuja MRF, Machado DA, Silveira MB, Debiaggi M, Reis MG, Cardoso RG, Blank P. Violência doméstica e suas diferentes manifestações. R Psiquiatr. 2003;25(Suppl 1):9-21.

33. Sedlak A, Mettenburg J, Basena M, Petta I, MacPherson K, Green A \& Li S. Fourth national incidence study of child abuse and neglect (NSI-4), 20092010: Report to Congress. U.S. Department of Health and Human Services (DHHS), Administration for Children and Families (ACF), Office of Planning, Research, and Evaluation (OPRE) and the Children's Bureau; 2010.

34. Diehl A, Cordeiro DC, Laranjeira R. Dependência química, prevenção, tratamento e políticas públicas. Porto Alegre: Artmed; 2011.

35. American Psychiatric Association. Manual Diagnóstico e Estatístico de Transtornos Mentais. Porto Alegre: Artmed; 1995.

36. Tavares BT, Bériab JU, Lima MS. Fatores associados ao uso de drogas entre adolescentes escolares. Rev Saúde Pública. 2004;38(6):787-96.

37. Pinto R, Ângela M. Dos maus-tratos na infância aos comportamentos de risco na idade adulta: um modelo conceptual. Congresso Luso-Brasileiro de Psicologia da Saúde. 2009 Feb 5-7. Faro, Portugal.

38. VIGITEL, 2012. Vigilância de fatores de risco e proteção para as doenças crônicas por inquérito telefônico. SUS/ Ministério da Saúde; 2013.

Recebido em: 26/10/2013

Aprovado em: 19/11/2013 